\title{
On chemically reacting hydromagnetic flow over a flat surface in the presence of radiation with viscous dissipation and convective boundary conditions
}

\author{
Emmanuel Maurice Arthur* , Ibrahim Yakubu Seini, Azizu Seidu \\ Mathematics Department, Faculty of Mathematical Sciences, Navrongo Campus, Ghana \\ Email address: \\ jimknights01@yahoo.com (E. M. Arthur), yakubuseini@yahoo.com (I. Y. Seini), abdulaziz_seidu@ @rocketmail.com (A. Seidu)
}

\section{To cite this article:}

Emmanuel Maurice Arthur, Ibrahim Yakubu Seini, Azizu Seidu. On Chemically Reacting Hydromagnetic Flow over a Flat Surface in the Presence of Radiation with Viscous Dissipation and Convective Boundary Conditions. American Journal of Applied Mathematics, Vol. 2, No. 5, 2014, pp. 179-185. doi: 10.11648/j.ajam.20140205.15

\begin{abstract}
This paper presents an investigation of the hydromagnetic flow over a flat surface with convective boundary conditions and internal heat generation in the presence of chemical reaction. The Newton-Raphson shooting method along with the fourth-order Runge-Kutta integration algorithm has been employed to tackle the third order, nonlinear boundary layer equation governing the problem. Results have been graphically displayed and discussed quantitatively to show some interesting aspects of the controlling parameters on the dimensionless axial velocity, temperature and the concentration profiles, local skin friction, and the rate of heat and mass transfer. Comparison of the numerical results of the present paper with earlier published works under some special cases showed consistency.
\end{abstract}

Keywords: Convective Boundary Condition, Hydrodynamics, Brinkmann Number, Viscous Dissipation, Heat Generation

\section{Introduction}

The study of hydrodynamic flow with heat and mass transfer over a flat plate may find its application to sheet extrusion in order to make flat plastic sheets. In doing so, it is important to investigate cooling and heat transfer for the improvement of the final products .The conventional fluids such as water and air are amongst the most widely used fluids as the cooling medium.

However, the rate of heat exchange achievable by the above fluids is realized to be unsuitable for certain sheet materials. Thus, in recent years, it has been proposed to alter flow kinematics so that, it will leads to a slower rate of solidification, as compared with water. Among the techniques to control flow kinematics, the idea of using magnetic fields appears to be the most attractive one, both because of its ease of implementation and also because of its non-intrusive nature. This has diverted the attention of researchers to the mathematical investigations of hydrodynamic flow of electrically conducting fluids with heat and mass transfer over surfaces with different orientations.

For instance, Unsteady MHD convective heat transfer past a semi-infinite vertical porous moving plate with variable suction was studied by Kim [10] whilst Chamkha and Khaled [5] investigated similarity solutions for hydromagnetic simultaneous heat and mass transfer by natural convection from an inclined plate with internal heat generation or absorption. In that same year, Seddeek [13] worked on thermal radiation and buoyancy effect on MHD free convection heat generation flow over an accelerating permeable surface with temperature dependent viscosity. Ouaf [12] did a numerical analysis of exact solution of thermal radiation on MHD flow over a stretching porous sheet. Ibrahim and Makinde [6] presented results on MHD boundary layer flow of chemically reacting fluid with heat and mass transfer past a stretching sheet.

Again, Ibrahim and Makinde [7] investigated into radiation effect on chemically reacting Magnetohydrodynamics (MHD) boundary layer flow of heat and mass transfer through a porous vertical plate. In that same year, Ibrahim and Makinde [8] presented a numerical analysis of chemically reacting Magnetohydrodynamics (MHD) boundary layer flow of heat and mass transfer past a low-heat-sheet moving vertically downwards. Heat and mass transfer by MHD mixed convection stagnation point flow toward a vertical plate embedded in a highly porous medium with radiation and internal heat generation was studied by Makinde [18]. Singh 
and Makinde [15] showed computational dynamics of MHD free convection flow along an inclined plate with Newtonian heating in the presence of volumetric heat generation whilst Makinde [11] studied entropy analysis for MHD boundary layer flow and heat transfer over a flat plate with a convective surface boundary condition. Alam et al [1] worked on heat and mass transfer in MHD free convection flow over an inclined plate with hall current whilst in that same year, Alia et al [2] presented the numerical results of radiation effects on MHD free convection flow along vertical flat plate in presence of Joule heating and heat generation. Alireza et al [3] also studied analytical Solution for Magnetohydrodynamic stagnation point flow and heat transfer over a permeable stretching sheet with chemical reaction. Ibrahim [9] investigated into heat and mass transfer effects on steady MHD flow over an exponentially stretching surface with viscous dissipation, heat generation and radiation. Seini and Makinde [14] studied MHD boundary layer flow due to exponential stretching surface with radiation and chemical reaction whilst Raja et al [16] presented result of Magneto-convection over a semi- infinite porous plate with heat generation.

Moreover, Uddin et al. [17] studied MHD forced convective laminar boundary layer flow from a convectively heated moving vertical plate with radiation and transpiration effect and recently, Arthur and Seini [4] studied MHD thermal stagnation point flow towards a stretching porous surface. This fact motivated the present communication to consider chemically reacting MHD flow over a flat surface in the presence of internal heat generation with convective boundary conditions

\section{Mathematical Model}

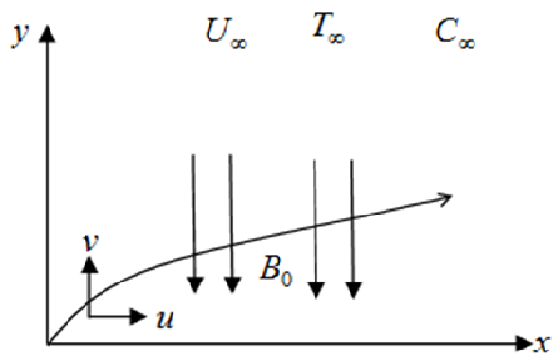

$$
T_{s} \quad C_{s} \quad u=v=0
$$

Figure 1. Schematic Diagram of the Problem

The steady laminar two-dimensional hydrodynamic boundary layer flow with heat and mass transfer over a flat plate in a stream of cold fluid at temperature $T_{\infty}$ in the presence of a volumetric rate of heat generation and magnetic field is considered.

It is assumed that the fluid property variations due to temperature and chemical species concentration are limited to fluid density.

It is also assumed that the lower surface of the plate is heated by convection from a hot fluid at temperature $T_{s}$ which provides the heat transfer coefficient $h_{s}$. The cold fluid on the upper side of the plate is assumed to be Newtonian and electrically conducting with constant fluid property. A uniform transverse magnetic field $B_{0}$ is imposed normal to the $x$-axis, as shown in Figure 1. Both the induced magnetic field due to the motion of the electrically conducting fluid and the electric field due to the polarisation of charges are assumed to be negligible.

Let the $x$-axis be taken along the direction of plate and $y$-axis, normal to it. If $u, v, T$ and $C$ are the fluid $x$-component of velocity, $y$-component of velocity, temperature and concentration respectively, then under the Boussinesq and boundary-layer approximations, and based on the above assumptions the continuity, momentum, energy and mass transfer (concentration) equations for the problem under consideration can be written as:

$$
\begin{gathered}
\frac{\partial u}{\partial x}+\frac{\partial v}{\partial y}=0 \\
u \frac{\partial u}{\partial x}+v \frac{\partial u}{\partial y}=v \frac{\partial^{2} u}{\partial y^{2}}-\frac{\sigma B_{0}^{2}}{\rho}\left(u-U_{\infty}\right) \\
u \frac{\partial T}{\partial x}+v \frac{\partial T}{\partial y}=\frac{\kappa}{\rho c_{p}} \frac{\partial^{2} T}{\partial y^{2}}+\frac{v}{c_{p}}\left(\frac{\partial u}{\partial y}\right)^{2}+ \\
\frac{\sigma B_{0}^{2}}{\rho c_{p}}\left(u-U_{\infty}\right)^{2}-\frac{1}{\rho c_{p}} \frac{\partial q_{r}}{\partial y} \\
u \frac{\partial C}{\partial x}+v \frac{\partial C}{\partial y}=D \frac{\partial^{2} C}{\partial y^{2}}-\gamma\left(C-C_{\infty}\right)
\end{gathered}
$$

Boundary conditions:

$$
\begin{gathered}
y=0: u=v=0,-\kappa \frac{\partial T}{\partial y}=h_{s}\left(T_{s}-T\right), \quad C=C_{s} \\
y \rightarrow \infty: u(x, y)=0, T=T_{\infty}, C=C_{\infty}
\end{gathered}
$$

where $v$ is the kinematic viscosity, $\sigma$ is the electrical conductivity, $T_{\infty}$ is the free stream temperature, $C_{\infty}$ is the free stream concentration, $U_{\infty}$ is the free stream velocity, $\alpha$ is the thermal diffusivity, $D$ is the mass diffusivity, $\kappa$ is the thermal conductivity, $B_{0}$ is the magnetic field of constant strength, $\rho$ is the fluid density, $c_{p}$ is the specific heat capacity at constant pressure, $\gamma$ is the reaction rate and $q_{r}$ is the radiative heat flux.

\section{Method of Solution}

The velocity components $u$ and $v$ can be expressed in terms of the stream function, $\psi(x, y)$ such that

$$
u=\frac{\partial \psi}{\partial y}, v=-\frac{\partial \psi}{\partial x}
$$

We seek for the stream function and the similarity variable respectively such that

$$
\psi(x, y)=x^{1 / 2} \sqrt{v U_{\infty}} f(\eta), \eta=x^{-1 / 2} y \sqrt{\frac{U_{\infty}}{v}}
$$


It may be verified that the continuity equation in (1), is identically satisfied.

Using the Rosseland approximation for radiation, Ibrahim and Makinde [7] simplified the heat flux as

$$
q_{r}=-\frac{4 \sigma^{*}}{3 K^{\prime}} \frac{\partial T^{4}}{\partial y}
$$

where $\sigma$ and $K^{\prime}$ are the Stefan-Boltzmann constant and the mean absorption coefficient respectively. We assume that the temperature differences within the flow such as the term $T^{4}$ may be expressed as a linear function of temperature. Hence, expanding $T^{4}$ in a Taylor series about $T_{\infty}$ and neglecting higher order terms, we get;

$$
T^{4} \cong 4 T_{\infty}^{3} T-3 T_{\infty}^{4}
$$

Also, the following non-dimensional temperature and concentration can be introduced as

$$
\theta(\eta)=\frac{T-T_{\infty}}{T_{S}-T_{\infty}}, \phi(\eta)=\frac{C-C_{\infty}}{C_{S}-T_{\infty}}
$$

Considering (6)-(10), we can transform (2)-(4) into the following ordinary nonlinear system of differential equations:

$$
\begin{gathered}
f^{\prime \prime \prime}(\eta)+\frac{1}{2} f(\eta) f^{\prime \prime}(\eta)-H a_{x}\left(f^{\prime}(\eta)-1\right)=0 \\
\left(1+\frac{4}{3} R a\right) \theta^{\prime \prime}(\eta)+\frac{1}{2} \operatorname{Pr} f(\eta) \theta^{\prime}(\eta)+ \\
B r f^{\prime \prime 2}(\eta)+B r H a_{x}\left(f^{\prime}(\eta)-1\right)^{2}=0 \\
\phi^{\prime \prime}(\eta)+\frac{1}{2} S c f(\eta) \phi^{\prime}(\eta)-S c \beta_{x} \phi(\eta)=0
\end{gathered}
$$

The corresponding boundary conditions in (3) now become

$$
\begin{gathered}
f(0)=0, f^{\prime}(0)=0, \theta^{\prime}(0)=B i_{x}[\theta(0)-1], \phi(0)=1, \\
f^{\prime}(\infty)=1, \theta(\infty)=0, \phi(\infty)=0 .
\end{gathered}
$$

In the above equations, primes denote the order of differentiation with respect to the similarity variable $\eta$, where $\operatorname{Pr}=\frac{v}{\alpha}$ (Prandtl number), $R a=\frac{4 \sigma^{*} T_{\infty}^{3}}{\kappa K^{\prime}}$ (Thermal radiation parameter), $H a_{x}=\frac{\sigma B_{0}^{2} x}{\rho U_{\infty}}$ (Local magnetic field parameter), $B r=\frac{\mu U_{\infty}^{2}}{\kappa\left(T_{w}-T_{\infty}\right)}($ Brinkmann number $), B i_{x}=\frac{h_{s}}{\kappa} \sqrt{\frac{v x}{U_{\infty}}}$ (Local Biot number), $S c=\frac{v}{D}$ (Schmidt number) and $\beta_{x}=\frac{\gamma x}{U_{\infty}}$ ( Local reaction rate parameter).

Obviously the local parameters $H a_{x}, B i_{x}$, and $\beta_{x}$ in (11)-(14) are functions of $x$. In order to have a similarity solution, all the parameters must be constant and we therefore assume that

$$
h_{s}=a x^{-1 / 2}, \sigma=b x^{-1} \text { and } \gamma=c x^{-1}
$$

where $a, b$ and $c$ are constants.

\section{Numerical Procedure}

The governing equations in (11)-(13) with the boundary conditions in (14) have been solved numerically by the use of Runge-Kutta integration along with the Newton Raphson algorithm to obtain approximate solutions. From the computations, the local skin friction coefficient, the local Nusselt number and the local Sherwood number at the plate surface which are respectively proportional to $-f^{\prime \prime}(0),-\theta^{\prime}(0)$ and $-\phi^{\prime}(0)$ are also worked out and their numerical values are presented in tabular form.

\section{Numerical Results}

The numerical results are compared with Aziz [19] and Makinde [11] in Table 1 to justify the accuracy of the method used. From the comparison, the present results are consistent with theirs.

In Table 2, it is observed that the rate of heat transfer, $-\theta^{\prime}(0)$, increases with increasing the Prandtl number and the local Biot number due to convective heat exchange at the surface of the plate. At the same time, the Lorenz force induced by increasing the magnetic field intensity increased the skin friction at the surface. Though this force is a retarding one, it enhanced the rate at which mass is transferred, $-\phi^{\prime}(0)$. Meanwhile this retarding force was distractive to the rate of heat transfer. Just as the magnetic field parameter, increasing the thermal radiation parameter and the Brinkmann number reduced the rate of heat transfer due to viscous dissipation. Furthermore, increasing the Schmidt number and the reaction rate parameter increases the rate of mass transfer for obvious reasons.

\subsection{Effects of Parameter Variation on the Velocity Profiles}

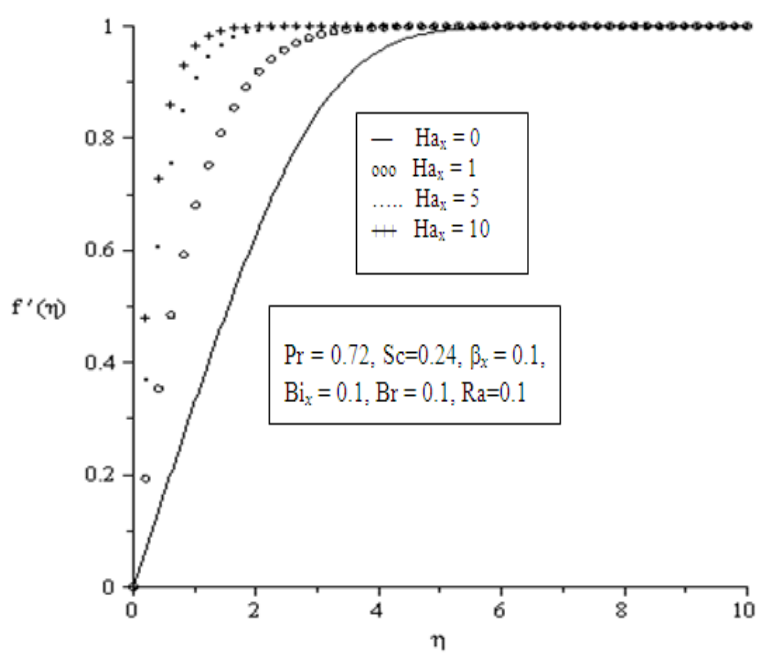

Figure 2. Velocity profiles for varying values of the magnetic parameter.

The effect of varying the controlling parameters on the velocity boundary layer is depicted in Figure 2. Generally, the fluid velocity is lowest at the plate surface and increases to the free stream value satisfying the far field boundary condition. A 
consistent decrease in the longitudinal velocity, informed by increasing magnetic field intensity, with all profiles tending asymptotically to the free stream value away from the plate, is observed. In practice, this phenomenon is due to the fact that increasing the magnetic field strength increases the Lorenz force which causes a greater opposition to fluid transport.

\subsection{Effects of Parameter Variation on the Temperature Profiles}

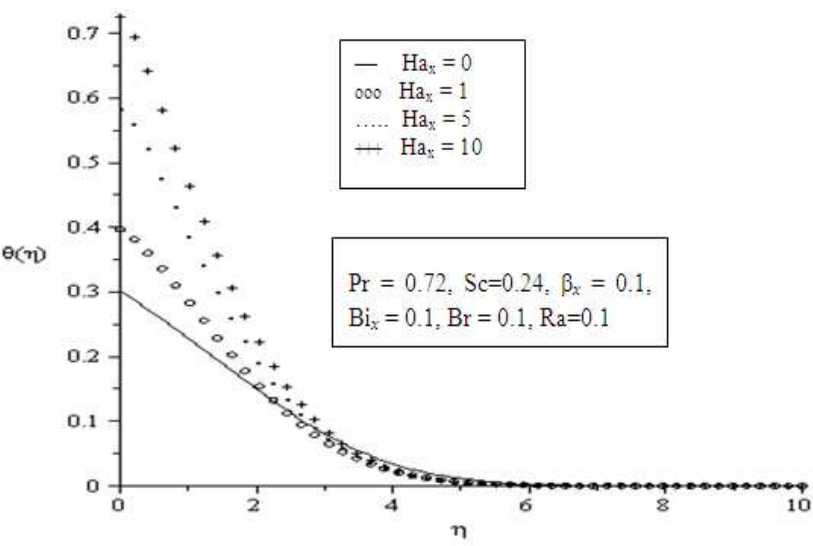

Figure 3. Temperature Profiles for varying local magnetic field parameter.

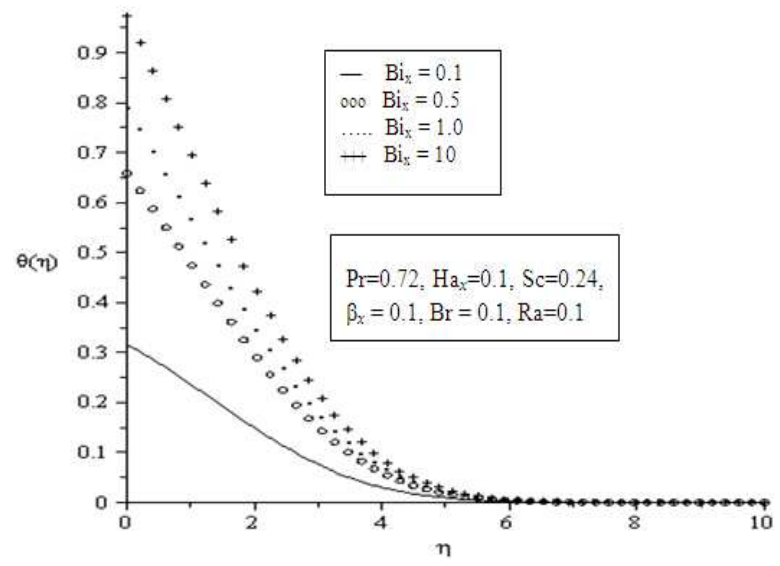

Figure 4. Temperature Profiles for varying local Biot number.

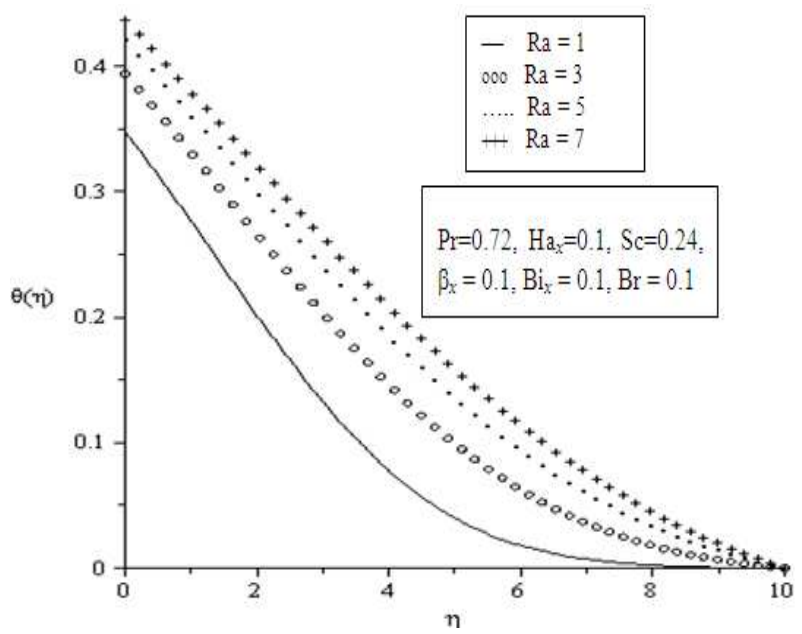

Figure 5. Temperature Profiles for varying radiation parameter.

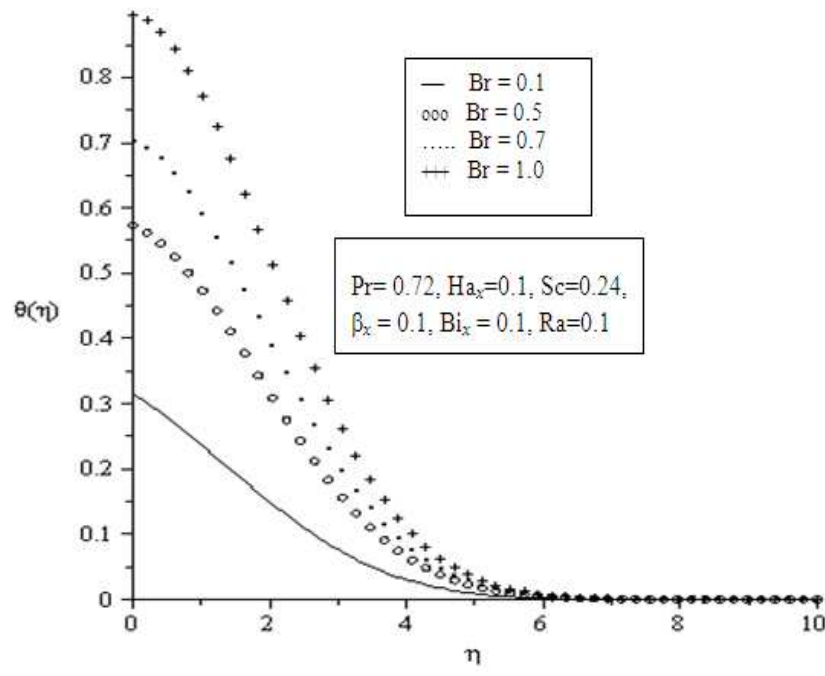

Figure 6. Temperature Profiles for varying Brinkmann number.

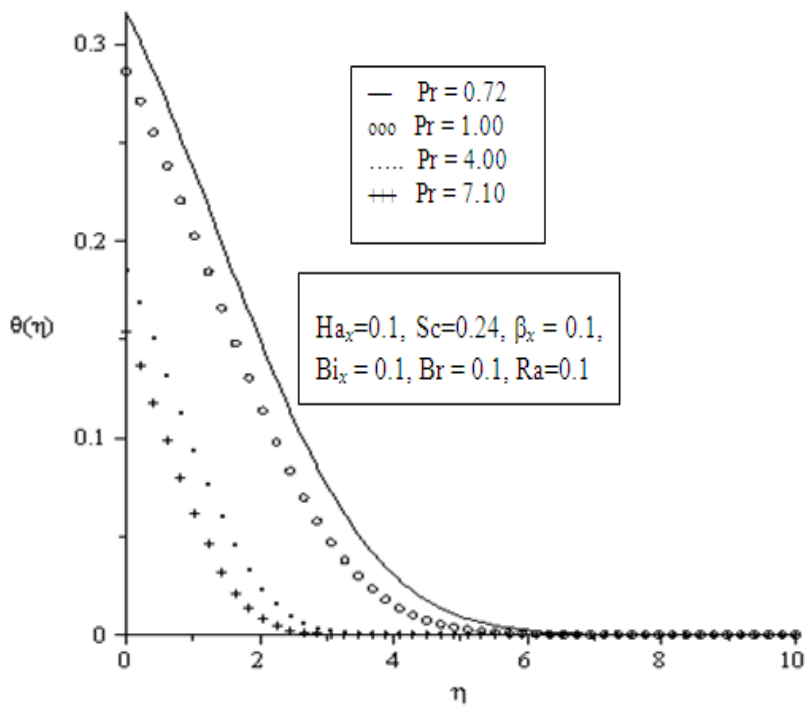

Figure 7. Temperature Profiles for increasing Prandtl number

Figures $3-7$ show the effects of the magnetic field parameter, Biot number, radiation parameter, Brinkmann number and Prandtl number respectively on the temperature profiles. It is observed that increasing the magnetic field intensity increases the fluid temperature which in turn, increases the thermal boundary layer. This can be attributed to the effect of ohmic heating on the flow system. An increase in the Biot number is observed to increase the temperature of the fluid due to the convective heat exchange between the hot fluid at the lower surface of the plate and the cold fluid at the upper surface of the plate. Increasing the thermal radiation causes an increase in the fluid temperature within the boundary layer. We observe the same trend for the Brinkmann number.

Meanwhile, increasing the Prandtl number decreases the fluid temperature within the boundary layer. When the Prandtl number is high, the fluid velocity decreases, which implies lower thermal diffusivity and hence, decrease in fluid temperature. 


\subsection{Effects of Parameter Variation of Concentration Profiles}

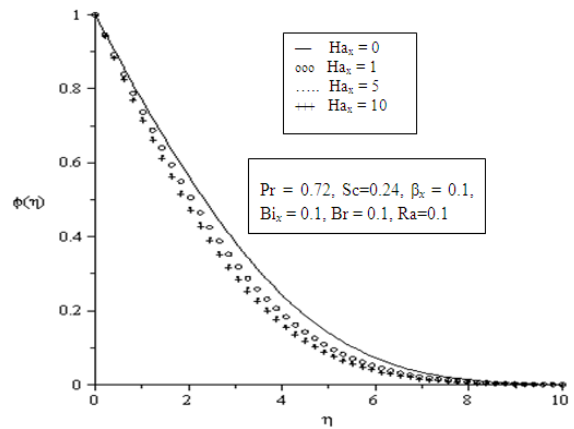

Figure 8. Concentration Profiles for increasing magnetic field parameter.

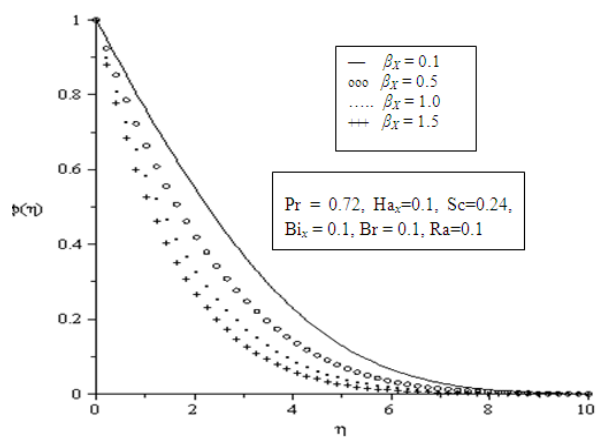

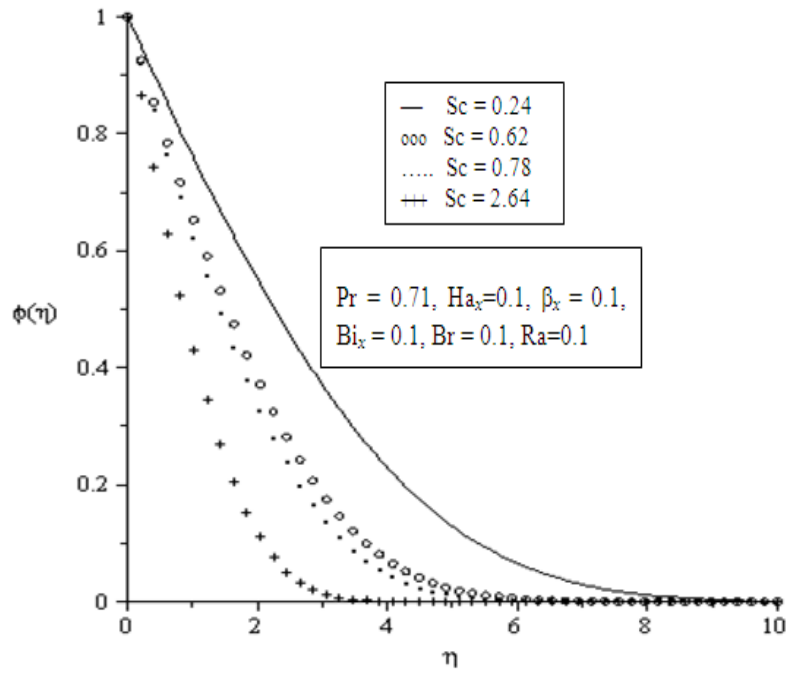

Figure 10. Concentration Profiles for increasing Schmidt number.

Figures $8-10$ show the effects of the Magnetic field parameter, reaction rate parameter and the Schmidt number respectively on the concentration boundary layer. It is observed that increasing the magnetic parameter reduces the species concentration boundary layer thickness. The same is true for increasing reaction rate parameter and the Schmidt number for obvious reasons.

Figure 9. Concentration Profiles for increasing local reaction rate parameter.

Table 1. Comparison of results for $\mathrm{Ha} a_{x}=0, \mathrm{Br}=0$ and $\mathrm{Pr}=0.72$

\begin{tabular}{|c|c|c|c|c|c|}
\hline \multicolumn{2}{|l|}{ Aziz [19] } & \multicolumn{2}{|c|}{ Makinde [11] } & \multicolumn{2}{|c|}{ Present Study } \\
\hline$-\theta^{\prime}(0)$ & $\theta(0)$ & $-\theta^{\prime}(0)$ & $\theta(0)$ & $-\theta^{\prime}(0)$ & $\theta(0)$ \\
\hline 0.0428 & 0.1447 & 0.04276 & 0.14466 & 0.042767 & 0.144661 \\
\hline 0.1981 & 0.6699 & 0.19805 & 0.66991 & 0.198051 & 0.669916 \\
\hline 0.2282 & 0.7718 & 0.22817 & 0.77182 & 0.228178 & 0.771822 \\
\hline 0.2791 & 0.9441 & 0.27913 & 0.94417 & 0.279131 & 0.944174 \\
\hline 0.2913 & 0.9854 & 0.29132 & 0.98543 & 0.291329 & 0.985434 \\
\hline
\end{tabular}

Table 2. Results of skin friction coefficient, Nusselt number and Sherwood number for various values of controlling parameters

\begin{tabular}{|c|c|c|c|c|c|c|c|c|c|}
\hline Pr & Sc & Hax & $\mathbf{R a}$ & $\mathrm{Br}$ & $\mathbf{B x}$ & Bix & $f^{\prime \prime}(\mathbf{0})$ & $-\theta^{\prime}(0)$ & $-\phi^{\prime}(\mathbf{0})$ \\
\hline 0.71 & 0.24 & 0.1 & 0.1 & 0.1 & 0.1 & 0.1 & 0.451835 & 0.068283 & 0.248586 \\
\hline 4.0 & 0.24 & 0.1 & 0.1 & 0.1 & 0.1 & 0.1 & 0.451835 & 0.081449 & 0.248586 \\
\hline 7.1 & 0.24 & 0.1 & 0.1 & 0.1 & 0.1 & 0.1 & 0.451835 & 0.084597 & 0.248586 \\
\hline 0.72 & 1.24 & 0.1 & 0.1 & 0.1 & 0.1 & 0.1 & 0.451835 & 0.068415 & 0.494321 \\
\hline 0.72 & 1.78 & 0.1 & 0.1 & 0.1 & 0.1 & 0.1 & 0.451835 & 0.068415 & 0.575143 \\
\hline 0.72 & 2.64 & 0.1 & 0.1 & 0.1 & 0.1 & 0.1 & 0.451835 & 0.068415 & 0.679036 \\
\hline 0.72 & 0.24 & 0.5 & 0.1 & 0.1 & 0.1 & 0.1 & 0.770792 & 0.064224 & 0.261862 \\
\hline 0.72 & 0.24 & 1.0 & 0.1 & 0.1 & 0.1 & 0.1 & 1.044009 & 0.060326 & 0.269658 \\
\hline 0.72 & 0.24 & 1.5 & 0.1 & 0.1 & 0.1 & 0.1 & 1.260190 & 0.057136 & 0.274372 \\
\hline 0.72 & 0.24 & 0.1 & 0.5 & 0.1 & 0.1 & 0.1 & 0.451835 & 0.066984 & 0.248586 \\
\hline 0.72 & 0.24 & 0.1 & 1.0 & 0.1 & 0.1 & 0.1 & 0.451835 & 0.065314 & 0.248586 \\
\hline 0.72 & 0.24 & 0.1 & 1.5 & 0.1 & 0.1 & 0.1 & 0.451835 & 0.063856 & 0.248586 \\
\hline 0.72 & 0.24 & 0.1 & 0.1 & 0.5 & 0.1 & 0.1 & 0.451835 & 0.042658 & 0.248586 \\
\hline 0.72 & 0.24 & 0.1 & 0.1 & 1.0 & 0.1 & 0.1 & 0.451835 & 0.010461 & 0.248586 \\
\hline 0.72 & 0.24 & 0.1 & 0.1 & 1.5 & 0.1 & 0.1 & 0.451835 & -0.021753 & 0.248586 \\
\hline 0.72 & 0.24 & 0.1 & 0.1 & 0.1 & 0.5 & 0.1 & 0.451835 & 0.068415 & 0.384277 \\
\hline 0.72 & 0.24 & 0.1 & 0.1 & 0.1 & 1.0 & 0.1 & 0.451835 & 0.068415 & 0.511822 \\
\hline 0.72 & 0.24 & 0.1 & 0.1 & 0.1 & 1.5 & 0.1 & 0.451835 & 0.068415 & 0.615465 \\
\hline 0.72 & 0.24 & 0.1 & 0.1 & 0.1 & 0.1 & 1 & 0.451835 & 0.209665 & 0.248586 \\
\hline 0.72 & 0.24 & 0.1 & 0.1 & 0.1 & 0.1 & 2 & 0.451835 & 0.236829 & 0.248586 \\
\hline 0.72 & 0.24 & 0.1 & 0.1 & 0.1 & 0.1 & 3 & 0.451835 & 0.247518 & 0.248586 \\
\hline
\end{tabular}




\section{Conclusion}

Chemically reacting MHD flow over a flat surface in the presence of internal heat generation with convective boundary conditions has been studied. Numerical results have been compared to earlier results published in the literature and consistency is shown. Our results revealed that:

i. A consistent decrease in the longitudinal velocity within the boundary layer accompanies a rise in the magnetic field intensity with all profiles tending asymptotically to the free stream value away from the plate.

ii. The thermal boundary layer increases with increasing values of the magnetic field parameter, Biot number, radiation parameter and Brinkmann number. Meanwhile, increasing the Prandtl number reduces it.

iii. The concentration boundary layer decreases with increasing Magnetic field parameter, reaction parameter and the Schmidt number.

iv. The skin friction at the surface increases for the increase in the magnetic field parameter.

v. The rate of heat transfer at the surface increases with increasing values of the Prandtl number and the Biot number; whereas a decrease is observed for increasing the magnetic field parameter, the radiation parameter and the Brinkmann number.

vi. The rate of mass transfer at the surface increases with increasing values of the magnetic field parameter, the reaction rate parameter and the Schmidt number.

\section{References}

[1] Alam, M. S., Ali, M., and Hossain, M. D., "Heat and Mass Transfer in MHD Free Convection Flow over an Inclined Plate with Hall Current", The International Journal Of Engineering And Science (IJES), 2(7), 2013, 81-88, ISSN (e): 2319 - 1813 ISSN (p): $2319-1805$

[2] Alia, M. M., Mamun, A. A., Maleque, Md. A., Hosain, N. and Azim, Md. A., "Radiation effects on MHD free convection flow along vertical flat plate in presence of Joule heating and heat generation", 5th BSME International Conference on Thermal Engineering. Procedia Engineering, 56, 2013, 503-509

[3] Alireza, R., Farzaneh-Gord, M., Varedi, S. R., Ganji, D., "Analytical Solution for Magnetohydrodynamic Stagnation Point Flow and Heat Transfer over a Permeable Stretching Sheet with Chemical Reaction", Journal of Theoretical and Applied Mechanics 51 (3) 2013, 675-686, Warsaw

[4] Arthur, E. M. and Seini, Y. I., "MHD Thermal Stagnation Point Flow towards a Stretching Porous Surface", Mathematical Theory and Modeling, 4(5)2014, 163-169, ISSN 2224-5804 (Paper) ISSN 2225-0522 (Online)

[5] Chamkha, A. J., and Khaled, A. R. A., "Similarity solutions for hydromagnetic simultaneous heat and mass transfer by natural convection from an inclined plate with internal heat generation or absorption”, Heat Mass Transfer, 37, 2001, 117-123
[6] Ibrahim, S. Y. and Makinde, D., "On MHD boundary layer flow of chemically reacting fluid with heat and mass transfer past a stretching sheet", International Journal of fluid Mechanics, 2(2) 2010, 123-132

[7] Ibrahim, S. Y. and Makinde, D., "Radiation effect on chemically reacting Magnetohydrodynamics (MHD) boundary layer flow of heat and mass transfer through a porous vertical plate", International Journal of Physical Sciences, 6(6), 2011, 1508-1516

[8] Ibrahim, S. Y. and Makinde, O. D., "Chemically reacting Magnetohydrodynamics (MHD) boundary layer flow of heat and mass transfer past a low-heat-sheet moving vertically downwards", Scientific Research and Essays, 6(22)2011, $4762-4775$

[9] Ibrahim, S.M., "Heat and Mass Transfer Effects on Steady MHD Flow over an Exponentially Stretching Surface with Viscous Dissipation, Heat Generation and Radiation”, Journal of Global Research in Mathematical Archives, 1(8) 2013.

[10] Kim, Y. J., "Unsteady MHD convective heat transfer past a semi-infinite vertical porous moving plate with variable suction”, Int. J. Eng. Sci., 38, 2000, 833-845

[11] Makinde, O.D., "Entropy analysis for MHD boundary layer flow and heat transfer over a flat plate with a convective surface boundary condition", Int. J. Exergy, 10(2)2012, pp. $142-154$.

[12] Ouaf, M. E. M., "Exact solution of thermal radiation on MHD flow over a stretching porous sheet", Appl. Math. Comput., 170, 2005, 1117-1125

[13] Seddeek, M. A., "Thermal Radiation and Buoyancy Effect on MHD Free Convection Heat Generation Flow over an Accelerating permeable Surface With temperature dependent viscosity", Can. J. Phys., 79, 2001, 725-732

[14] Seini, Y. I. and Makinde, O. D., "MHD boundary layer flow due to exponential stretching surface with radiation and chemical reaction", Mathematical Problems in Engineering, 2013.

[15] Singh, G. and Makinde, O. D., "Computational Dynamics of MHD Free Convection Flow along an inclined plate with Newtonian heating in the presence of Volumetric heat Generation", Chemical Engineering Communication, 199(9)2012, 1144-1154.

[16] Raja, T., Karthikeyan, S. and Senthilnathan, B., "A Magneto-convection Over a Semi- infinite Porous Plate with Heat Generation". Journal of Applied Fluid Mechanics, 6 (4)2013, pp. 589-595

[17] Uddin, M. J., Khan, W. A., and Ismail, A. I. M., "MHD Forced Convective Laminar Boundary Layer Flow from a Convectively Heated moving Vertical Plate with Radiation and Transpiration Effect", PLoS ONE 8(5)2013,e62664. doi:10.1371/journalpone.0062664.

[18] Makinde, O. D., "heat and mass transfer by MHD mixed convection stagnation point flow toward a vertical plate embedded in a highly porous medium with radiation and internal heat generation", An International Journal of Theoretical and Applied Mechanics. Meccanica (2012)47, 2011,1173-1184, DOI 10.1007/s11012-011-9502-5 
[19] Aziz, A., "A similarity solution for laminar thermal boundary layer over a flat plate with a convective surface boundary condition", Commun. Nonlinear Sci. Numer Simulat., Vol. 14, 2009, pp. 1064-1068 